\title{
L. On the variation of the semi-axis major of the moon's orbit
}

\author{
John William Lubbock Esq. Treas. R.S. F.R.A.S. F.L.S.
}

To cite this article: John William Lubbock Esq. Treas. R.S. F.R.A.S. F.L.S. (1840) L. On the variation of the semi-axis major of the moon's orbit , Philosophical Magazine Series 3, 17:111, 338-346, DOI: $10.1080 / 14786444008650189$

To link to this article: http://dx.doi.org/10.1080/14786444008650189

册 Published online: 01 Jun 2009.

Submit your article to this journal $[\pi$

Џll Article views: 3

Q View related articles $\square$ 
L. On the I'ariation of the Semi-axis Major of the Moon's Orbit. By John Wrldiam Lubbock, Esq., Treas. R.S. F.R.A.S. and F.L.S., Vice.Chancellor of the University of London, \&c.

DOISSON, in his Mémoire sur le Mouvement de la Lune autour de la Terre, has considered the following theorem, that the expression for the variation of the semi-axis major contains no argument of long period, accompanied by a multiple of $m$ less than $m^{4}$. In this paper he has taken into account the terms which arise from the second approximation, or that in which the squares and products of the quantities $\delta \zeta, \delta a, \delta e, \delta \omega, \delta \gamma$ and $\delta \alpha$ may be neglected. It is evident that terms may arise in the next, and indeed in every succeeding approximation of the order $m^{3}$, which must be taken into account in order to prove the proposition with sufficient generality. Thus the variation of the eccentricity contains terms multiplied by $m$, as of the argument $9,(2 \tau-2 \xi)$; these, multiplied by others of the order $m^{2}$, give in $\delta e^{2}$ terms of the order $m^{3}$, and these multiplied by $\frac{\mathrm{d}^{3} R}{\mathrm{~d} e^{2} \mathrm{~d} c}$ give in $\frac{\mathrm{d} R}{\mathrm{~d} c} \mathrm{~d} t$ terms multiplied by $m^{5}$, which after integration become of the order $m^{3}$; if the argument being of the kind under consideration, the divisor introduced by integration is of the order $m^{2}$. It is true that Poisson refers to his Mémoire sur la Variation des Constants arbitraires Mém. de l'Academie, tom. i., for an extension to the third approximation, that is, to terms depending upon the cube of the disturbing force. In this paper, however, expressions are employed, which take for granted that the disturbing force can be exhibited in the same form, developed in terms of the initial values of the coordinates $x, y, z$, and of the initial values of their first differential coefficients $\frac{\mathrm{d} x}{\mathrm{~d} t}, \frac{\mathrm{d} y}{\mathrm{~d} t}, \frac{\mathrm{d} z}{\mathrm{~d} t}$. This development has never been executed, nor has it been shown to be possible. When this system of constants is employed, the quantities which are equivalent to $[a, \omega]$, $[a, e]$, \&c., become equal to unity and rigorously constant, so that it is unnecessary to consider the effect produced by their variation. Even, however, with the simplifications which recourse to this peculiar system of constants affords, Poisson admits that this demonstration would become too complicated to admit of its extension to the higher powers of the disturbing force. M. de Pontécoulant has made objections to the proof given by Poisson, but as he differentiates* in a manner at variance with that intended in the expressions of which Poisson makes use, and does not allude to the Memoir in question, in which a further approximation is attempted, these objections are not to be considered as exactly identical with those of that distinguished astronomer.

\footnotetext{
* Conn. des Temps, 1840, p.21.
} 
In the Lunar Theory terms of the order $m^{3}$, and of the nature of those under consideration, may arise not only in the third but in every succeeding approximation, and hence it becomes absolutely necessary to seek some mode of proof which admits of unlimited extension, and I have therefore endeavoured to modify the proof given by Poisson, so as to include all terms of the order $\mathrm{m}^{3}$, however far the approximation be pushed, and without introducing any peculiar system of constants.

For this purpose it is necessary to suppose the disturbing function $R$

$$
\begin{aligned}
& =(R)+\left(\frac{\mathrm{d} R}{\mathrm{~d} \zeta}\right) \delta \zeta+\left(\frac{\mathrm{d} R}{\mathrm{~d} a}\right) \delta a+\left(\frac{\mathrm{d} R}{\mathrm{~d} c}\right) \delta c \\
& +\left(\frac{\mathrm{d} R}{\mathrm{~d} e}\right) \delta e+\left(\frac{\mathrm{d} R}{\mathrm{~d} \omega}\right) \delta \omega+\left(\frac{\mathrm{d} R}{\mathrm{~d} \gamma}\right) \delta \gamma+\left(\frac{\mathrm{d} R}{\mathrm{~d} a}\right) \delta \alpha \\
& \zeta=n t \quad \quad \frac{\mathrm{d} R}{\mathrm{~d} \zeta}=\frac{\mathrm{d} R}{\mathrm{~d} c} \quad n^{2} a^{y}=\mu ;
\end{aligned}
$$

and to define $\delta \zeta, \delta a, \delta c, \& c$, to represent, not the total variation of the quantities $\zeta, a, c, e, \& c$., as in Poisson's paper, but that portion only which consists of arguments corresponding to those in-equalities which are not depressed by integration, as will be presently explained. The effect of the secular inequalities of the constants $c, \omega$ and $\alpha$, and also that of all the other inequalities of which the arguments are independent of $c$, are supposed to be already included in the quantities

$$
(R),\left(\frac{\mathrm{d} R}{\mathrm{~d} a}\right), \quad\left(\frac{\mathrm{d} R}{\mathrm{~d} c}\right), \& c .
$$

As the constant $c$ in Poisson's notation always accompanies $n t$, all the arguments in the development of $R$ may be properly represented by an expression of the form

$$
i(n t+c)+j m t+l t+\beta
$$

where $i$ and $j$ are whole numbers or zero; $l$ a certain multiple of $m^{2}$ depending upon the secular variation of the angles $c, \omega, a$, and $\beta$ a quantity rigorously constant, which accompanies $l t$, but which I shall in future omit to write down. Whenever $i=0$ without $j$ becoming $=0$ at the same time, the corresponding inequality in the expressions for the elliptic constants is necessarily a multiple of $m$ at least, and only when $i$ and $j$ are both equal to zero the corresponding inequality in each of those expressions may no longer be multiplied by $m$.

$$
\mathrm{d} a=[a, c] \frac{\mathrm{d} R}{\mathrm{~d} c} \mathrm{~d} t+[a, \omega] \frac{\mathrm{d} R}{\mathrm{~d} \omega} \mathrm{d} t+\& \mathrm{c} .
$$




$$
\mathrm{d} c=[c, a] \frac{\mathrm{d} R}{\mathrm{~d} a} \mathrm{~d} t+[c, \omega] \frac{\mathrm{d} R}{\mathrm{~d} \omega} \mathrm{d} t+\& \mathrm{c} .
$$

The quantities between brackets may be considered as constant in the first approximation, and such that

$$
[c, a]=-[a, c] \quad[\omega, c]=-[c, \omega] .
$$

Similar theorems exist with respect to all the constants, and it is upon this property that the theorem in question may be said to depend. If $a, e, \gamma, c, \omega, \alpha$, have the same signification as in Poisson's memoir (Mémoire de l'Institut, tom. xiii.)

$$
\begin{array}{lll}
{[a, e]=0} & {[a, \gamma]=0} & {[e, \gamma]=0} \\
{[e, \omega]=0} & {[c, \gamma]=0} & {[\omega, \alpha]=0 ;}
\end{array}
$$

but these latter theorems do not appear to influence the proposition of which the proof is required.

In differentiating the disturbing function with regard to $c$, as indicated in the expression

$$
\mathrm{d} a=-\frac{2 \mathrm{~d} R}{a n \mathrm{~d} c} \mathrm{~d} t
$$

$a$ being the semi-axis major, $R$ must be differentiated only with respect to $c$, inasmuch as it was contained in $R$ primitively, and not as it is introduced in further approximations by the variations of the elliptic elements. As, however, the secular variations and those multiplied by $m$ and $m^{0}$, which $I$ have already included in

$$
(R), \quad\left(\frac{\mathrm{d} R}{\mathrm{~d} a}\right),\left(\frac{\mathrm{d} R}{\mathrm{~d} e}\right), \& c .
$$

do not contain $i$ or $c$, and as therefore $c$ only occurs in the quantities

$$
\text { (R), }\left(\frac{\mathrm{d} R}{\mathrm{~d} a}\right), \quad\left(\frac{\mathrm{d} R}{\mathrm{~d} e}\right), \quad \& \mathrm{c} .,
$$

as it was introduced primitively *,

$$
\begin{aligned}
\frac{\mathrm{d} R}{\mathrm{~d} c} & =\frac{\mathrm{d}(\boldsymbol{R})}{\mathrm{d} c}+\left(\frac{\mathrm{d}^{2} R}{\mathrm{~d} c^{2}}\right) \delta \zeta+\left(\frac{\mathrm{d}^{2} \boldsymbol{R}}{\mathrm{d} a \mathrm{~d} c}\right) \delta a+\left(\frac{\mathrm{d}^{2} R}{\mathrm{~d} c^{3}}\right) \delta c \\
& +\left(\frac{\mathrm{d}^{2} \boldsymbol{R}}{\mathrm{d} e \mathrm{~d} c}\right) \delta c+\left(\frac{\mathrm{d}^{2} R}{\mathrm{~d} \omega \mathrm{d} c}\right) \delta \omega+\left(\frac{\mathrm{d}^{2} R}{\mathrm{~d} \gamma \mathrm{d} c}\right) \delta \gamma+\left(\frac{\mathrm{d}^{2} R}{\mathrm{~d} a \mathrm{~d} c}\right) \delta \alpha
\end{aligned}
$$

$\delta \zeta, \delta a, \delta e, \& c$. , denoting here only that portion of the variations of $\zeta, a, e, \& \mathrm{c}$., which consists of periodical terms not independent of $i$, and therefore multiplied by the square of $m$ at least.

* This remark applies solely to the constant $c$, and to no other. 
the Semi-axis Major of the Moon's Orbit.

$$
\delta \zeta=-\frac{3 n}{2 a} \int \delta a \mathrm{~d} t
$$

and it is sufficient to take

$$
\begin{aligned}
& \frac{\mathrm{d} a}{\mathrm{~d} t}= \frac{1}{a^{2} n}\left(3 n \frac{\mathrm{d}^{2} R}{\mathrm{~d} c^{2}} \int \delta a \mathrm{~d} t-\frac{\mathrm{d} R}{\mathrm{~d} c} \delta a\right) \\
&-2\left\{\left(\frac{\mathrm{d}^{2} R}{\mathrm{~d} a \mathrm{~d} c}\right) \frac{\delta a}{a n}+\left(\frac{\mathrm{d}^{2} R}{\mathrm{~d} c^{2}}\right) \frac{\delta c}{a n}+\frac{\mathrm{d}^{2} R}{\mathrm{~d} e \mathrm{~d} c} \frac{\delta e}{a n}\right. \\
& \\
&\left.+\left(\frac{\mathrm{d}^{2} R}{\mathrm{~d} \omega \mathrm{d} c}\right) \frac{\delta \omega}{a n}+\left(\frac{\mathrm{d}^{2} R}{\mathrm{~d} \gamma \mathrm{d} c}\right) \frac{\delta \gamma}{a n}+\left(\frac{\mathrm{d}^{2} R}{\mathrm{~d} a \mathrm{~d} c}\right) \frac{\delta \alpha}{a n}\right\} .
\end{aligned}
$$

This equation is similar in appearance only to the equation $(A)$ of Poisson, p. 259, for the quantities $\delta a, \delta c, \delta e, \& c$., are here differently defined.

I omit here all consideration of the terms

$$
\frac{1}{a^{2} n}\left(3 n \frac{\mathrm{d}^{2} R}{\mathrm{~d} c^{2}} \int \delta a \mathrm{~d} t-\frac{\mathrm{d} R}{\mathrm{~d} c} \delta a\right),
$$

because the proof which Poisson has given in his Mémoire sur le Mouvement de la Lune, is completely satisfactory with reference to them.

In the terms $\frac{\delta c}{a n}, \frac{\delta e}{a n}, \& \mathrm{c}$. , it is necessary to consider the variation of the quantities $[a, w],[e, w], \& c$, , and also that of the quantity $\left(\frac{1}{a n}\right)$

$$
\mathrm{d} \cdot \frac{1}{a n}=-\frac{\mathrm{d} a}{n a^{2}}-\frac{\mathrm{d} n}{n a^{2}}=\frac{\mathrm{d} a}{2 n a^{2}}=-\frac{\mathrm{d} R}{\mu \mathrm{d} c} \mathrm{~d} t ;
$$

$\mathrm{d} e$ contains the term

$$
[e, \omega] \frac{\mathrm{d} R}{\mathrm{~d} \omega} \mathrm{d} t,
$$

which may be taken as a type of others;

$$
\begin{aligned}
\frac{1}{a n} \int[e, \omega]\left(\frac{\mathrm{d} R}{\mathrm{~d} \omega}\right) \mathrm{d} t=\int \frac{[e, \omega]}{a n}\left(\frac{\mathrm{d} R}{\mathrm{~d} \omega}\right) \mathrm{d} t \\
+\mathrm{d} \cdot \frac{1}{a n} \int[e, \omega]\left(\frac{\mathrm{d} R}{\mathrm{~d} \omega}\right) \mathrm{d} t \\
=\int \frac{[e, \omega]}{a n}\left(\frac{\mathrm{d} R}{\mathrm{~d} \omega}\right) \mathrm{d} t-\frac{\mathrm{d} R}{\mu \mathrm{d} c} \int[e, \omega]\left(\frac{\mathrm{d} R}{\mathrm{~d} \omega}\right) \mathrm{d} t .
\end{aligned}
$$


It must be recollected that in this expression no terms are included in $\delta e$ or $\int[e, \omega] \frac{\mathrm{d} R}{\mathrm{~d} t}$ which are independent of $c$.

If $\left(\frac{\mathrm{d} R}{\mathrm{~d} e}\right)$, as defined and limited in the expression for $\boldsymbol{R}$ in p. 339 , contains any term

$$
A \cos (i(n t+c)+j m n t+l n t),
$$

$\mathrm{d} \omega$ contains the term

$$
[a, \omega] A \cos (i(n t+c)+j m n t+l n t)
$$

and considering now only the constant portion of $[a, \omega], \delta \omega$ contains the term

$$
[a, \omega] \frac{A}{(i+j m+l) n} \sin (i(n t+c)+j m n t+l n t) .
$$

Similarly, if $\left(\frac{\mathrm{d} R}{\mathrm{~d} \omega}\right)$ contains the term

$$
B \sin \left(i(n t+c)+j m n t+l^{\prime} n t\right)
$$

$\mathrm{d} e$ contains the term

$$
[\omega, e] B \sin \left(i(n t+c)+j m n t+l^{\prime} n t\right),
$$

$\delta e$ contains the term

$$
-[\omega, e] \frac{B}{\left(i+j m+l^{\prime}\right) n} \cos \left(i(n t+c)+j m n t+l^{\prime} n t\right),
$$

and these terms give, after well-known reductions, in $\int \frac{\mathrm{d} R}{\mathrm{~d} c} \mathrm{~d} t$

$$
[\omega, e] \frac{i A B}{n(i+j m+l)(i+j m+l)} \cos (l-l) t,
$$

which is evidently of the order $m^{4}, A$ and $B$ being each of the order $m^{2}$, and $i$ of necessity not equal to zero. tity

I now proceed to consider the effect of the variation of the quan.

$$
\frac{[e, \omega]}{a n},
$$

which may be taken as the type of other similar quantities.

Let

$$
\frac{[e, \omega]}{a n}=C+D \cos (i n t+j m n t+l n t)
$$


the Semi-axis Major of the Moon's Orbit.

$$
\begin{aligned}
& \left(\frac{\mathrm{d} R}{\mathrm{~d} e}\right)=A \cos \left(i^{\prime} n t+j^{\prime} m n t+l^{\prime} n t\right) \\
& \frac{[e, \omega]}{a n}\left(\frac{\mathrm{d} R}{\mathrm{~d} e}\right)=C A \cos \left(i^{\prime} n t+j m n t+l^{\prime} n t\right) \\
& \quad+\frac{D A}{2} \cos \left(i^{\prime} n t-i n t+j^{\prime} m n t-j m n t+l^{\prime} n t-l n t\right) \\
& \quad+\frac{D A}{2} \cos \left(i^{\prime} n t+i n t+j^{\prime} m n t+j m n t+l^{\prime} n t+l n t\right) .
\end{aligned}
$$$$
\text { Let }\left(\frac{\mathrm{d} R}{\mathrm{~d} \omega}\right)=B \sin \left(i^{\prime \prime} n t+j^{\prime \prime} m n t+l^{\prime \prime} n \dot{t}\right)
$$

$$
\begin{aligned}
& \frac{[e, \omega]}{a n}\left(\frac{\mathrm{d} R}{\mathrm{~d} \omega}\right)=C B \sin \left(i^{\prime \prime} n t+j^{\prime \prime} m n t+l^{\prime \prime} n t\right) \\
& \quad+\frac{D B}{2} \sin \left(i^{\prime \prime} n t-i n t+j^{\prime \prime} m n t-j m n t+l^{\prime \prime} n t-l n t\right) \\
& \quad+\frac{D B}{2} \sin \left(i^{\prime \prime} n t+i n t+j^{\prime \prime} m n t+j m n t+l^{\prime \prime} n t+l n t\right) .
\end{aligned}
$$

$$
\begin{aligned}
& \int \frac{[e, \omega]}{a n}\left(\frac{\mathrm{d} R}{\mathrm{~d} \omega}\right) \mathrm{d} t \\
&=-C B \frac{\cos \left(i^{\prime \prime} n t+j^{\prime \prime} m n t+l^{\prime \prime} n t\right)}{\left(i^{\prime \prime}+j^{\prime \prime} m+l^{\prime \prime}\right) n} \\
&-\frac{D B}{2} \frac{\cos \left(i^{\prime \prime} n t-i n t+j^{\prime \prime} m n t-j m n t+l^{\prime \prime} n t-l n t\right)}{\left(i^{\prime \prime}-i+j^{\prime \prime} m-j m+t^{\prime \prime}-l\right) n} \\
&-\frac{D B}{2} \frac{\cos \left(i^{\prime \prime} n t+i n t+j^{\prime \prime} m n t+j m n t+l^{\prime \prime} n t+l n t\right)}{\left(i^{\prime \prime}+i+j^{\prime \prime} m+j m+l^{\prime \prime}+l\right) n}
\end{aligned}
$$$$
-\int[e, \infty] \frac{\mathrm{d} R}{\mathrm{~d} e} \mathrm{~d} t
$$$$
=-C A \frac{\sin \left(i^{\prime} n t+j^{\prime} m n t+l^{\prime} n t\right)}{\left(i^{\prime}+j^{\prime} m+l^{\prime}\right) n}
$$$$
-\frac{D A}{2} \frac{\sin \left(i^{\prime} n t-i n t+j^{\prime} m n t-j m n t+l^{\prime} n t-\ln t\right)}{\left(i^{\prime}-i+j^{\prime} m-j m+l^{\prime}-l\right) n}
$$$$
-\frac{D A}{2} \frac{\sin \left(i^{\prime} n t+i n t+j^{\prime} m n t+j m n t+l^{\prime} n t+l n t\right)}{\left(i^{\prime}+i+j^{\prime} m+j m+l^{\prime}+l\right) n}
$$

$\left(\frac{\mathrm{d}^{2} R}{\mathrm{~d} e \mathrm{~d} c}\right)=-i^{\prime} A \sin \left(i^{\prime} n t+j^{\prime} m n t+l \cdot n t\right)$ 
344 Mr. Lubbock on the Variation of

$$
\left(\frac{\mathrm{d}^{2} R}{\mathrm{~d} \omega \mathrm{d} c}\right)=i^{\prime \prime} B \cos \left(i^{\prime \prime} n t+j^{\prime \prime} m n t+l^{\prime \prime} n t\right) \text {. }
$$

These terms give in $\frac{\mathrm{d} a}{\mathrm{~d} t}$

and therefore

$$
\left(\frac{\mathrm{d}^{2} R}{\mathrm{~d} e \mathrm{~d} c}\right) \frac{\delta e}{a n}+\frac{\mathrm{d}^{2} R}{\mathrm{~d} \omega \mathrm{d} c} \frac{\delta \omega}{a n},
$$

$$
\left(\frac{\mathrm{d}^{2} R}{\mathrm{~d} e \mathrm{~d} c}\right) \int \frac{[e, \omega]}{a n}\left(\frac{\mathrm{d} R}{\mathrm{~d} \omega}\right) \mathrm{d} t+\left(\frac{\mathrm{d}^{2} R}{\mathrm{~d} \omega \mathrm{d} c}\right) \int \frac{[\omega, e]}{a n}\left(\frac{\mathrm{d} R}{\mathrm{~d} e}\right) \mathrm{d} t
$$

and hence the following terms :

$$
i^{\prime} C B A \sin \left(i^{\prime} n t+j^{\prime} m n t+l^{\prime} n t\right)
$$

$$
\begin{gathered}
\frac{\cos \left(i^{\prime \prime} n t+j^{\prime \prime} m n t+l^{\prime \prime} n t\right)}{\left(i^{\prime \prime}+j^{\prime \prime} m+l^{\prime \prime}\right) n} \\
+\frac{i^{\prime} D B A}{2} \sin \left(i^{\prime} n t+j^{\prime} m n t+l^{\prime} n t\right) \\
\frac{\cos \left(i^{\prime \prime} n t-i n t+j^{\prime \prime} m n t-j m n t+l^{\prime \prime} n t-l n t\right)}{\left(i^{\prime \prime}-i+j^{\prime \prime} m-j m+l^{\prime \prime}-l\right) n} \\
+\frac{i^{\prime} D B A}{2} \sin \left(i^{\prime} n t+j^{\prime} m n t+l^{\prime} n t\right) \\
\frac{\cos \left(i^{\prime \prime} n t+i n t+j^{\prime \prime} m n t+j m n t+l^{\prime \prime} n t+l n t\right)}{\left(i^{\prime \prime}+i+j^{\prime \prime} m+j m+l^{\prime \prime}+l\right) n} \\
-i^{\prime \prime} C B A \cos \left(i^{\prime \prime} n+j^{\prime \prime} m n t+l^{\prime \prime} n t\right) \\
-\frac{\sin \left(i^{\prime} n t+j^{\prime} m n t+l^{\prime} n t\right)}{\left(i^{\prime}+j^{\prime} m+l^{\prime}\right) n} \\
\frac{i^{\prime \prime} D B A}{2} \cos \left(i^{\prime \prime} n t+j^{\prime \prime} m n t+l^{\prime \prime} n t\right) \\
-\frac{\sin \left(i^{\prime} n t-i n t+j^{\prime} m n t-j m n t+l^{\prime} n t-l^{\prime} n t\right)}{\left(i^{\prime}-i+j^{\prime} m-j^{\prime \prime}+l^{\prime}-l\right) n} \\
\frac{i^{\prime \prime} D A}{2} \cos \left(i^{\prime \prime} n t+j^{\prime \prime} m n t+l^{\prime \prime} n t\right) \\
\frac{\sin \left(i^{\prime} n t+i n t+j^{\prime} m n t+j m n t+l^{\prime} n t+l n t\right)}{\left(i^{\prime}+i+j^{\prime} m+j^{\prime \prime} n+l^{\prime}+l\right) n} .
\end{gathered}
$$

The terms multiplied by $C B A$, which arise from the constant portion of $[e, \omega]$, have already been considered, the rest form four 
pairs, of which the following is one, and may be taken as a type of the rest:

$\frac{D B A}{4 n}\left\{\frac{i^{\prime}}{i^{\prime \prime}-i+j^{\prime \prime} m-j m+l^{\prime \prime}-l}-\frac{i^{\prime \prime}}{i^{\prime}+i+j^{\prime} m+j m+l^{\prime}+l}\right\}$

$\sin \left(i^{\prime} n t+j^{\prime} m n t+l^{\prime} n t-i^{\prime \prime} n t+i n t-j^{\prime \prime} m n t+j m n t-l^{\prime \prime} n t+l n t\right)$.

In order that this argument may be of the kind under consideration, we must have

$$
\begin{aligned}
& i^{\prime}-i^{\prime \prime}+i=0 \\
& j^{\prime} m-j^{\prime \prime} m+j m=0
\end{aligned}
$$

and the term becomes in that case

$$
\begin{aligned}
& \frac{D B A}{4 n}\left\{\frac{i^{\prime}}{i^{\prime \prime}-i+j^{\prime \prime} m-j m+l^{\prime \prime}-l}\right. \\
&\left.-\frac{i^{\prime \prime}}{i^{\prime}+i+j^{7} m+j m+l^{\prime}+l}\right\} \sin \left(l^{\prime}-l^{\prime \prime}+l\right) n t
\end{aligned}
$$

if $i$ is not equal to zero, $D$ is of the order $m^{2}$, and the coefficient of $\sin \left(l^{\prime}-l^{\prime \prime}+l\right) n t$, after a fresh integration, remains of the order $m^{4}$. If $i=0$

$$
i^{\prime}=i^{\prime \prime}
$$

the coefficient becomes

$$
\begin{aligned}
& \frac{i^{\prime} D B A}{4 n}\left\{\frac{j^{\prime} m+j m+l^{\prime}+l-j^{\prime \prime} m+j m-l^{\prime \prime}+l}{\left(i^{\prime \prime}+j^{\prime \prime} m-j m+l^{\prime \prime}-l\right)\left(i^{7}+j^{7} m+j m+l^{\prime}+l\right)}\right\} \\
& =\frac{i^{\prime} D B A\left\{j m+l^{\prime}-l^{\prime \prime}+22 l\right\}}{\left.4 n\left\{i^{\prime \prime}+j^{\prime \prime} m-j m+l^{\prime \prime}-l\right)\left(i^{\prime}+j^{\prime} m+j m+l^{\prime}-l\right)\right\}} ;
\end{aligned}
$$

but unless $j=0, D$ is multiplied by $m$, and in either case, in consequence of the reductions which the numerator undergoes, the coefficient after a fresh integration remains of the order $m^{4}$.

It remains now only to consider the terms of which the following is the type:

$$
\left(\frac{\mathrm{d}^{2} R}{\mathrm{~d} e \mathrm{~d} c}\right) \frac{\mathrm{d} R}{\mathrm{~d} c} \int[e, \omega]\left(\frac{\mathrm{d} R}{\mathrm{~d} \omega}\right),
$$

but as in $\delta e$ are here only included terms of the order $m^{2}$,

$$
\int[e, \omega]\left(\frac{\mathrm{d} R}{\mathrm{~d} \omega}\right)
$$


is of the order $m^{2}$, or at least such portion of it as is supposed tobeincluded in this expression; and as $\frac{\mathrm{d}^{2} R}{\mathrm{~d} e \mathrm{~d} c}$ and $\frac{\mathrm{d} R}{\mathrm{~d} c}$ are each of the order $m^{2}$, this term is of the order $m^{6}$, which after a fresh integration remains of the order $m^{4}$.

Hence we may conclude with safety, without having recourse to any peculiar system of constants, or to any precarious induction, that however far the approximation be carried, the variation of the semi-axis major in the Lunar Theory contains no term of long period multiplied by a power of $m$ inferior to the fourth.

Sept. 21, 1840.

LI. Some Observations on the aqueous Solution of Carbonate of Magnesia with excess of Carbonic Acid, and on the Salt which it affords by spontaneous Decomposition. By JoHN DAvY, M.D., F.R.S.*

I

HAVE been induced to institute some experiments on the solution of carbonate of magnesia in water strongly inpregnated with carbonic acid gas, in consequence of the high repute, on very questionable grounds, which it has lately acquired as a medicine.

The solution I have used is that prepared and sold by $\mathrm{Mr}$. Dinneford of New Bond-street, with the designation of Dinneford's Solution of Magnesia, and with the following recommendation on the label: "The great advantages of this elegant preparation are, that being in a fluid state and possessing all the properties of magnesia in general use, it is not likely to form dangerous concretions in the bowels; it corrects acidity and heart-burn effectually, without injuring the coats of the stomach, as carbonates of potash and soda are known to do; it prevents the food of infants turning sour, and in all cases it acts as a pleasing aperient, particularly adapted for females."

Such a recommendation $I$ should not have thought it right to notice, were it an ordinary quack eulogy, and unsupported by certificates given by respectable medical men ; and moreover were I not assured that great faith is placed by many persons in the asserted virtues of the preparation, and that the use of it is rapidly extending.

The first trials I subjected the medicine to, were made with a view to test the permanence of the solution; as by ex-

* Communicated by Sir David Brewster. 